\title{
Los Objetivos de Desarrollo Sostenible en el plan de estudios del Grado en Ingeniería Civil de la Universitat Politècnica de València
}

\author{
M. Esther Gómez-Martín ${ }^{a}$, Ester Gimenez-Carbo ${ }^{b}$, Ignacio Andrés-Doménech ${ }^{c}$ \\ ${ }^{a}$ Universitat Politècnica de València, Valencia, España, mgomar00@upv.es, ${ }^{b}$ Universitat Politècnica \\ de València, Valencia, España, esgimen@cst.upv.es, Universitat Politècnica de València, Valencia, \\ España, igando@hma.upv.es.
}

\section{Resumen}

En septiembre de 2015 la Asamblea General de la ONU adoptó la Agenda 2030 para el Desarrollo Sostenible, en la que se plantean 17 objetivos con 169 metas interdependientes sobre cuestiones económicas, ambientales y sociales. Es el acuerdo internacional más ambicioso hasta la fecha y es necesaria la colaboración de todos los actores de la sociedad para hacer frente a los desafios que en ella se plantean.

La Universidad tiene que reflexionar acerca del servicio que presta a la sociedad y adaptarse a las nuevas necesidades del siglo XXI, permaneciendo en contacto con la ciudadanía y contribuyendo a la resolución de los problemas, desafios y conflictos de nuestro tiempo. Por ello, adoptar el contenido de la Agenda 2030 como marco de trabajo, ayudará a integrar en los estudios universitarios los Objetivos de Desarrollo Sostenible (ODS).

La presente comunicación recoge el trabajo desarrollado en la Escuela Técnica Superior de Ingeniería de Caminos, Canales y Puertos de Valencia para adaptar el plan de estudios del Grado en Ingeniería Civil con objeto de integrar los ODS en los resultados del aprendizaje del título. Así, los estudiantes incorporarán a su formación la adquisición de habilidades y competencias que les permitan desarrollar en su desempeño profesional como ingenieros soluciones sostenibles.

Para ello se ha realizado un diagnóstico del plan de estudios y se han identificado las asignaturas que contribuyen a cada uno de los 17 ODS. Con esta información se han establecido los contenidos a incorporar para mejorar la formación de los ingenieros civiles para alcanzar las metas de la Agenda 2030. 
Palabras claves: ODS, Agenda 2030, Ingeniería Civil, Plan de Estudios.

\section{Introducción}

\subsection{La Agenda 2030 en España}

El 25 de septiembre de 2015 la asamblea general de la Organización de las Naciones Unidas (ONU, 2015) adoptó uno de los acuerdos globales más ambiciosos y transcendentes de nuestro tiempo: la Agenda 2030 para el Desarrollo Sostenible con el lema "transformando nuestro mundo". Es un plan de acción a favor de las personas, el planeta y la prosperidad que también tiene la intención de fortalecer la paz universal y el acceso a la justicia.

La Agenda plantea 17 Objetivos de Desarrollo Sostenible (ODS) con 169 metas de carácter integrado e indivisible que pretende abordar los desafíos mundiales más acuciantes: acabar con la pobreza y promover la prosperidad económica, la inclusión social, la sostenibilidad medioambiental, la paz y el buen gobierno para todos los pueblos para el 2030.

En junio de 2018 el gobierno de España aprobó el "Plan de acción para la implementación de la Agenda 2030" (Gobierno de España, 2018) en la que se presenta el estado del país, analizando la situación de los ODS en España y se abordan las acciones a llevar a cabo para poner en marcha la Agenda. En este documento se menciona a la Universidad como actor imprescindible que debe comprometerse para la implementación de la Agenda y se indican explícitamente 7 contribuciones de las universidades españolas a la aplicación de la Agenda 2030. Entre ellas queremos destacar:

a. (2) Un compromiso decidido con la inclusión de competencias relacionadas con un desarrollo sostenible e inclusivo, necesarias para la construcción de una ciudadanía global, en la formación de todo el estudiantado, el personal docente e investigador y el personal de administración y servicios.

b. (3) La generación y la transferencia de un conocimiento comprometido con el desarrollo sostenible, incluyendo aquí también el conocimiento necesario para articular y dar seguimiento a la propia Agenda 2030.

c. (7) Compromiso por parte de las universidades, a reportar informes acerca de sus impactos en términos de docencia, investigación y transferencia, alineándose a cada uno de los ODS."

En este contexto, la Universitat Politècnica de València, a través del Vicerrectorado de Calidad y Acreditación, está llevando a cabo un programa de innovación docente para alinear el perfil competencial de sus egresados con los ODS, mediante su incorporación en 
los resultados del aprendizaje. En primera instancia, la UPV exigirá a partir del curso 2019/2020 que el estudiante incorpore a su Trabajo de Fin de Título, una reflexión explícita que justifique en qué medida el trabajo se relaciona con determinados ODS. Además, se ofrecerá a los estudiantes formación on-line especializada y específica sobre cada uno de los ODS. En una segunda fase, se incentivará la incorporación de los ODS en las propias titulaciones, a todos los niveles. Es en este último contexto en el que se centra el trabajo que se presenta.

\subsection{La profesión de la ingeniería civil y los ODS}

En las sociedades actuales, la actividad profesional ocupa un lugar significativo teniendo una clara influencia en la estructuración y funcionamiento de la vida social. Por ello es de vital importancia para la cimentación de sociedades comprometidas con la Agenda 2030, que los profesionales conozcan y asuman los ODS en sus quehaceres diarios.

Asimismo, la profesión de la ingeniería civil tiene una gran responsabilidad como motor que transforma el medio, y desde la creación de la primera Institución de ingenieros civiles (ICE) en Reino Unido, se ha reflexionado sobre el trabajo que realizan. En 1828 Tredgold (Ashley, 2012)en el momento de la creación de la institución definió la ingeniería civil como "el arte de dirigir las grandes fuentes de poder de la Naturaleza para el uso y conveniencia del hombre, como medio de producción y de tráfico en los estados, tanto para el comercio exterior como para el interior, tal como se aplica en la construcción de carreteras, puentes, acueductos, canales, navegación fluvial y muelles, para el intercambio y el intercambio interno; y en la construcción de puertos, topos, rompeolas y faros, y en el arte del a navegación con energía artificial, para fines comerciales; y en la construcción y adaptación de maquinaria y en el desagüe de ciudades y pueblos". Esta definición se ha ido transformando con el paso del tiempo y en el año 2007 esta institución la reformuló de la siguiente manera: "la ingeniería civil es un arte vital, que trabaja con las grandes fuentes de poder de la naturaleza para la riqueza y el bienestar de toda la sociedad".

Las asociaciones de ingeniería civil de todo el mundo definen la profesión en términos similares y por ejemplo la American Society of Civil Engineers (ASCE) de Estados Unidos dice que "los ingenieros civiles diseñan, construyen y mantienen los cimientos de nuestras sociedades modernas - nuestras carreteras y puentes, el agua potable y los sistemas de energía, los puertos y los aeropuertos, y la infraestructura para un medio ambiente más limpio".

Esta asociación a partir de La Cumbre sobre el Futuro de la Ingeniería Civil celebrada en junio de 2006, publicó el documento "La visión para la ingeniería civil en 2025" (ASCE, 2007), en el que se articula una visión global de esta profesión y se muestran las tareas a las 
que deben aspirar los ingenieros del siglo XXI: "En 2025, los ingenieros civiles prestarán servicio de manera competente, colaborativa y ética como maestros:

- Planificadores, diseñadores, constructores y operarios del motor económico y social de la sociedad: el medio ambiente construido;

- custodios del medio ambiente natural y sus recursos;

- innovadores e integradores de ideas y tecnología en los sectores público, privado y académico;

- gestores de los riesgos y las incertidumbres causados por acontecimientos naturales, accidentales y otras amenazas; y

- líderes en debates y decisiones que conformarán la política pública ambiental y de infraestructuras".

En general todas las asociaciones de ingenieros civiles del mundo toman estas definiciones como propias para explicar las tareas de la profesión y queda clara su trascendencia en la trasformación del medio, en la construcción de redes de transporte, y en el abastecimiento de recursos, lo que conlleva una gran responsabilidad y una influencia directa en la consecución de la agenda 2030. Por todo ello es necesario que desde las escuelas de ingeniería civil se promueva y se instruya a los estudiantes para conseguir los ODS.

\section{Cómo empezar}

\subsection{Método}

Para estudiar cómo integrar los ODS en el curriculum de los ingenieros civiles se puede tomar como punto de partida los esfuerzos llevados a cabo en diversas universidades para introducir la sostenibilidad en sus planes de estudio (Holmberg et al. 2008, Lozano 2010 and Watson et al. 2013). En los trabajos mencionados se emplean diversas metodologías para abordar la tarea de analizar los planes de estudio. En este trabajo, para abordar la tarea de integrar los ODS en el curriculum de los ingenieros civiles se ha seguido una metodología basada en la herramienta STAUNCH (Lozano, 2010). Para ello será necesario identificar los contenidos relacionados que ya se están impartiendo, identificando a que ODS contribuyen, se señalaran las asignaturas que pueden contribuir y se indicaran nuevos contenidos que pueden introducirse en las actividades que se desarrollan en la Escuela para que los alumnos adquieran de manera holística las competencias y conocimientos que aborda la agenda 2030 . 


\subsection{Diagnóstico}

Una vez revisado el plan de estudios se ha comprobado que, como se intuía al iniciar la tarea, el curriculum que siguen los estudiantes para convertirse en ingenieros civiles tiene un gran potencial para integrar contenidos relacionados directamente con los ODS.

El análisis detallado de las guías docentes de las 59 asignaturas que conforman el plan de estudios ha puesto de manifiesto que actualmente solo hay 17 asignaturas que trabajen contenidos directamente relacionados con alguna de las 169 metas de los 17 ODS. Estas 17 asignaturas se concentran en 11 de los 17 ODS. Destacando principalmente los siguientes ODS:

- ODS \#6 “Agua limpia y saneamiento", trabajado en 6 asignaturas correspondientes a los cursos de $1^{\circ}, 3^{\circ}$ y $4^{\circ}$.

- ODS \#9 “Industria, innovación e infraestructura", trabajado en 4 asignaturas correspondientes a los cursos de $2^{\circ}$ y $3^{\circ}$.

- ODS \#11 “Ciudades y comunidades sostenibles", trabajado en 5 asignaturas correspondientes a los cursos de $3^{\circ}$ y $4^{\circ}$.

- ODS \#13 “Acción por el clima”, trabajado en 3 asignaturas correspondientes a los cursos de $3^{\circ}$ y $4^{\circ}$.

Sin embargo, este análisis ha puesto de manifiesto, por un lado, que las asignaturas que ya integran alguno de los ODS en sus contenidos, podrían integrar también otros ODS y, por otro lado, que hay 25 asignaturas que están relacionadas con los ODS y aunque no los trabajan en la actualidad, se podrían implementar de forma más o menos sencilla, ya que sus contenidos están vinculados a algunas de las metas definidas en los 17 ODS. Finalmente, solo existen 17 asignaturas de las 59 que integran el plan de estudios que no se han podido relacionar con ninguno de los ODS, las cuales fundamentalmente se corresponden con las materias de formación básica de matemáticas, física y dibujo.

A la vista de las metas relacionadas directamente con cada asignatura se ha demostrado que la ingeniería civil está esencialmente relacionada con 5 de los 17 ODS, los cuales se consideran los ODS más vinculados con la titulación y por tanto los que pueden ser trabajados en mayor número de asignaturas, estos son:

- ODS \#6 Agua limpia y saneamiento: en 10 asignaturas.

- ODS \#9 Industria, innovación e infraestructura: en 25 asignaturas

- ODS \#11 Ciudades y comunidades sostenibles: en 15 asignaturas

- ODS \#12 Producción y consumo responsables: en 16 asignaturas

- ODS \#13 Acción por el clima: en 12 asignaturas

El resto de los ODS se pueden trabajar en distinto número de asignaturas (entre 1 y 9) con diferente profundidad y alcance según el caso. Además, todos los ODS se pueden trabajar 
en otras actividades universitarias que dan lugar al reconocimiento de créditos contemplados en el RD1393/2007.

Las asignaturas con mayor potencial para trabajar los ODS, teniendo en cuenta el número de ODS que se trabajan o se pueden llegar a trabajar son las asignaturas siguientes:

- Transporte y territorio ( $2^{\circ}$ curso): 5 ODS

- Hidráulica e hidrología (3er curso): 6 ODS

- Obras marítimas ( $3^{\text {er }}$ curso): 6 ODS

- Gestión de empresas ( $4^{\circ}$ curso): 5 ODS

- Infraestructuras hidráulicas ( $4^{\circ}$ curso): 6 ODS

- Gestión de empresas consultoras y constructoras ( $4^{\circ}$ curso): 5 ODS

- Ética en la ingeniería civil ( $4^{\circ}$ curso): 8 ODS

La asignatura de $4^{\circ}$ curso Ingeniería civil para la sociedad tiene una importancia relevante ya que es la única asignatura del plan de estudios vinculada con el ODS \#16 Paz, justicia e instituciones solidarias. Del mismo modo, la asignatura Ética en la ingeniería civil, es la única asignatura que desarrolla el ODS \#4.

En el siguiente apartado se va a realizar una propuesta con acciones concretas.

\section{Propuesta}

\subsection{Acciones en tres niveles}

La incorporación de mejoras en una titulación en la Universitat Politècnica de València incluyen, atendiendo al Manual de Calidad de la institución, mejoras en dos esferas: mejoras en los procesos propios de la Universitat o de la Escuela y mejoras en el propio diseño del título.

Con este contexto, se plantean acciones en tres niveles diferentes para la incorporación de los ODS en los resultados del aprendizaje y perfil competencial de los egresados del Grado en Ingeniería Civil.

El primer nivel, de aplicación inmediata, consiste en introducir contenidos relacionados con la Agenda 2030 de manera transversal, para que todos los estudiantes conozcan su contenido. Para los autores, el modo más inmediato de conseguirlo es planteando una actividad formativa obligatoria para los alumnos de primer curso y que se puede impartir en el marco del Programa de Acción Tutorial (PATU) (Giménez-Carbó et al., 2017). Esta actividad estará orientada a explicar los objetivos perseguidos por la Agenda 2030, los 17 
ODS y las 169 metas. Este nivel se corresponde con las mejoras en los procesos propios, en este caso, de la Escuela, mencionados anteriormente.

El segundo nivel planteará cambios a nivel asignatura. Una vez realizado el análisis explicado en el apartado 2, se tiene conocimiento de las asignaturas relacionadas con los ODS. Por lo que se propone mantener reuniones con los profesores responsables de dichas materias, junto con expertos en Agenda 2030, y abordar cambios para que, en los resultados del aprendizaje de los alumnos, estén presentes los ODS. Este nivel se corresponde con propuestas de mejora que no suponen una modificación de la memoria de verificación del título. En consecuencia, su implantación es relativamente sencilla y viable.

El tercer nivel se corresponde con propuestas de mejora que suponen una modificación de la memoria de verificación del título. El margen de acción y la inmediatez en la implantación de estas propuestas es muy reducido. No obstante, conviene plantear también acciones en este nivel pues son las que pueden dar lugar a cambios más integrados y articulados en todo el plan de estudios.

Todas las potencialidades detectadas en el diagnóstico se pueden abordar con actuaciones del segundo nivel, dado que simplemente pasarían por incorporar acciones específicas en las guías docentes de las asignaturas. Por otra parte, en futuras revisiones del plan de estudios que dieran lugar a modificaciones en la memoria de verificación podría plantearse a tenor del diagnóstico realizado, si asignaturas actualmente optativas que trabajan directamente os ODS, como Ingeniería civil para la sociedad o ética en la ingeniería civil no debieran pasar a formar parte del currículo obligatorio.

\subsection{Validación de la eficacia de las acciones.}

Además de estudiar como introducir los ODS en el plan de estudios de los ingenieros civiles, también nos teníamos que plantear como verificar que los estudiantes alcanzaban estos resultados de aprendizaje. Todos los alumnos deben superar exámenes para certificar que han adquirido los conocimientos impartidos en cada materia, así que los contenidos relacionados con los ODS que se introducían en cada asignatura son relativamente fáciles de evaluar (por supuesto será necesario diseñar correctamente los actos de evaluación para que esta afirmación sea válida). Pero también es muy importante saber que la integración de estos contenidos en el currículo ha hecho que estén presentes en cada trabajo que los ingenieros civiles desarrollen en su vida profesional.

Será muy difícil llevar un seguimiento del trabajo de los egresados una vez abandonen la Escuela (en este momento será responsabilidad de los colegios profesionales), pero si se puede evaluar si están presentes en el trabajo fin de grado, el último acto que los estudiantes 
realizan para conseguir la titulación y que en muchos casos es muy similar a los trabajos técnicos que realizaran durante su carrera profesional.

Por ello, se ha desarrollado una rúbrica, para analizar la capacidad de los estudiantes para incorporar los ODS en su trabajo. Esta rúbrica se ha diseñado teniendo en cuenta las 5 partes en que se agrupan los ODS y se han planteado 4 niveles (A, B, C, D) de consecución de integración del ODS, pero en dos alcances, potencial y asignado. La adaptación de la rúbrica a 4 niveles se ha realizado para mantener el mismo sistema de calificación que se lleva a cabo en el UPV para evaluar las competencias transversales presentes en todas sus titulaciones. Esta rúbrica también estará a disposición de los profesores de las asignaturas para que puedan evaluar la capacidad de implementación de los ODS en los trabajos entregados por los estudiantes en cada asignatura concreta.

\section{Conclusiones}

Una vez estudiado y analizado el plan de estudios del Grado en Ingeniería Civil se puede concluir:

a. En el caso de la formación de los ingenieros civiles es imprescindible que los ODS estén presentes en su plan de estudios.

b. Se ha mostrado una metodología sencilla en la que a partir de la revisión de las guías docentes de las asignaturas que conforman las distintas materias del plan de estudios, se han detectado la presencia y las potencialidades de incorporación de los ODS en la formación de los estudiantes.

c. Se han expuesto acciones a tres niveles para incorporar los ODS en el currículo.

d. Y por último se ha indicado como validar la adquisición de estos resultados de aprendizaje a nivel de asignatura y a nivel de Trabajo Fin de Grado, diseñando una rúbrica para facilitar la evaluación.

\section{Agradecimientos}

Los autores agradecen a la UPV, en específico al VECA (Convocatoria A+D. Proyectos de Innovación y Mejora Educativa), como entidad financiadora del proyecto.

\section{Referencias}

ONU Organización Naciones Unidas (2015). Objetivos de Desarrollo Sostenible. Recuperado de https://www.un.org/sustainabledevelopment/es/2015/09/ 
Gobierno de España (2018). Plan de acción para la implementación de la Agenda 2030. Hacia una estrategia española de desarrollo sostenible. Recuperado el 18 de mayo de 2019, de http://www.exteriores.gob.es/Portal/es/SalaDePrensa/Multimedia/Publicaciones

Ashley, R. (2012). The role of the civil engineer in society: engineering ethics and major projects. Proceedings of the Institution of Civil Engineers, 165(3), 99.

ASCE. (2007). The vision for civil engineering in 2025. Proc., Summit on the Future of Civil Engineering. Recuperado el 1 de mayo de 2019, de https://www.asce.org/uploadedFiles/About_Civil_Engineering/Content_Pieces/vision2025.pdf

Holmberg, J., Svanström, M., Peet, D. J., Mulder, K., Ferrer-Balas, D., \& Segalàs, J. (2008). Embedding sustainability in higher education through interaction with lecturers: Case studies from three European technical universities. European Journal of Engineering Education, 33(3), 271-282.

Lozano, R. (2010). Diffusion of sustainable development in universities' curricula: an empirical example from Cardiff University. Journal of Cleaner Production, 18(7), 637-644.

Watson, M. K., Lozano, R., Noyes, C., \& Rodgers, M. (2013). Assessing curricula contribution to sustainability more holistically: Experiences from the integration of curricula assessment and students' perceptions at the Georgia Institute of Technology. Journal of Cleaner Production, 61, 106-116. 